\title{
Creación y análisis de un repositorio videográfico educativo para la sensibilización de problemas sociales en la educación superior
}

\author{
Celia Corchuelo-Fernández ${ }^{1 *}$, Aránzazu Cejudo-Cortes ${ }^{1}$, Esteban Vázquez-Cano² ${ }^{2}$ Eloy López-Meneses ${ }^{3,4}$ \\ (1) Universidad de Huelva, Facultad de Educación, Huelva-España. (correo-e: celia.corchuelo@dedu.uhu.es; \\ carmen.cejudo@dedu.uhu.es) \\ (2) Universidad Nacional de Educación a Distancia (UNED), Facultad de Educación Ciencias Sociales, Madrid-España. \\ (correo-e: evazquez@edu.uned.es) \\ (3) Universidad Pablo de Olavide, Facultad de Ciencias Sociales. Sevilla-España. (correo-e: elopmen@upo.es) \\ (4) Instituto de Investigaciones en Ciencias Sociales y Educación, Universidad de Atacama, Copayapu \# 485, Copiapó, \\ Chile
}

${ }^{*}$ Autor a quien debe ser dirigida la correspondencia.

Recibido Dic. 26, 2019; Aceptado Feb. 24, 2020; Versión final Abr. 25, 2020, Publicado Oct. 2020

\begin{abstract}
Resumen
Este artículo se centra en el análisis de 76 vídeos elaborados por 357 estudiantes de diferentes titulaciones en los años 2013 a 2018, que conforman un repositorio videográfico creado por el alumnado, para concienciar y sensibilizar a la ciudadanía sobre los actuales problemas sociales. Este estudio está enmarcado dentro del Proyecto Innovación Docente 2.0 Tecnologías de la Información y la Comunicación en el Espacio Europeo de Educación Superior, desarrollado por la Universidad Pablo de Olavide (España). La metodología ha sido cuantitativa/cualitativa, analizando las temáticas más recurrentes y la calidad en la ejecución de los vídeos por medio del diseño y validación de una rúbrica. Las frecuencias temáticas muestran que los estudiantes ven prioritario dar respuestas educativas y sociales al acoso escolar e inmigración, entre otros. Se concluye que las tecnologías emergentes son recursos valiosos para la construcción de conocimientos en los procesos de enseñanza-aprendizaje, propiciando la reformulación de metodologías sociocontructivistas e investigadoras.
\end{abstract}

Palabras clave: educación superior; vídeos educativos; innovación pedagógica; educación social; evaluación del aprendizaje

\section{Creation and analysis of an educational video repository to raise awareness about social issues in higher education}

\begin{abstract}
This article focuses on the analysis of 76 videos produced by 357 students from different degrees during the years 2013 to 2018, which consists of a video repository created by the students, to raise awareness and sensitize citizens about current social issues. This study is framed within the Project Teaching Innovation 2.0 Information and Communication Technologies in the European Higher Education Area that was developed by the University Pablo de Olavide (Spain). The methodology is quantitative and qualitative. A rubric was designed and validated to analyze the videos' execution quality and most recurrent themes. The thematic frequencies show that students see priority in providing educational and social responses to school bullying and immigration. It is concluded that emerging technologies are valuable resources for the construction of knowledge in the teaching-learning processes to promote the reformulation of socio-constructivist and research methodologies.
\end{abstract}

Keywords: higher education; educational video; pedagogical innovation; social education; learning assessment 


\section{INTRODUCCIÓN}

Este estudio surge de la directriz marcada por el proyecto Innovación docente 2.0 con Tecnologías de la Información y la Comunicación en el Espacio Europeo de Educación Superior, desarrollado en el marco de los Proyectos de Innovación y Desarrollo Docente de la Universidad Pablo de Olavide (UPO), financiado por el Vicerrectorado de Docencia y Convergencia Europea de dicha Universidad y al amparo de los investigadores del grupo de investigación EDUINNOVAGOGÍA (HUM-971), reconocido por el Plan Andaluz de Investigación, Desarrollo e Innovación y la Oficina de Transferencia de Resultados de Investigación de la Universidad Pablo de Olavide. Con el propósito de innovar en el ámbito de la educación se buscan estrategias y metodologías novedosas, motivantes y beneficiosas que faciliten al estudiante su aprendizaje, que aumenten su interés e implicación. Se pretenden, por una parte, encontrar técnicas que atraigan e involucren al alumno para que logren buenos resultados. Y por otra, que los estudiantes identifiquen y utilicen estrategias apropiadas a las diferentes tareas y situaciones de aprendizaje y así promover la transferencia de aprendizajes a otros contextos. De ahí la preocupación por implementar buenas prácticas docentes que produzcan cambios y fomenten la obtención de resultados competenciales (Abarca y Soto, 2014).

El profesor es responsable de realizar una buena práctica educativa, cambiando su metodología que pasará de una práctica tradicional a una innovadora, generadora de cambios positivos en la escuela y el alumnado. Es la buena práctica la que transforma los procesos educativos o implanta otros nuevos, desembocando en una innovación metodológica óptima y enriquecedora para los estudiantes. Por este motivo las prácticas no deben de ser rígidas, ni venir determinadas, deben ajustarse a la realidad en la que están inmersas, respetando sus contextos y moldeándose a ellos. Desde este criterio es el profesor el que debe de autoevaluarse y saber que cambiar, reformular y mejorar en su práctica docente. Este ejercicio ayuda al docete a interiorizar que debe continuar formándose para estar a la altura de las exigencias de la sociedad del conocimiento al incrementar el nivel de rendimiento, el nivel competencial y mejorar los resultados académicos del estudiante que tiene en sus aulas.

La sociedad del conocimiento nos proporciona una gran cantidad de información, creada y extinguida rápidamente. Corremos el riesgo de no poder digerirla o no tener tiempo para hacerlo, de ahí la necesidad de poder seleccionar aquella útil y práctica para nuestra formación y desarrollo profesional. Esto nos lleva a tener que generar conocimientos pertinentes que nos permitan resolver problemas que se presenten. Eso es precisamente lo que pretenden los diseños por competencias: que las personas desarrollen capacidades amplias, que les permitan aprender, y desaprender, a lo largo de toda su vida para adecuarse a situaciones cambiantes. Además, este cambio acelerado del saber, es cada vez más complejo, obedeciendo a una lógica posmoderna que nos cuesta articular porque equiparamos complejidad y complicación. Hay que eliminar la fragmentación y apostar por un saber integral. Las competencias constituyen una clara apuesta en esta línea, proponiendo la movilización de conocimientos y su combinación pertinente para responder a situaciones en contextos diversos. Las propuestas por competencias incluirían el conjunto de conocimientos, habilidades y actitudes de carácter muy diferente, incorporando talentos o inteligencias que tradicionalmente desde los sistemas educativos reglados no se habían tenido presentes.

Se entienden las competencias como la capacidad efectiva para llevar a cabo exitosamente una actividad laboral plenamente identificada. Las competencias serian el conjunto de conocimientos, procedimientos y actitudes combinados, coordinados e integrados en la acción, adquiridos a través de la experiencia (formativa y no formativa) que permite al individuo resolver problemas específicos de forma autónoma y flexible en contextos singulares. Se precisa que los estudiantes sean capaces de interpretar el contexto social y profesional vinculado a sus estudios universitarios y sean capaces de diseñar actuaciones que supongan una mejora de los mismos. En este sentido, el educador social es un profesional que realiza una acción socioeducativa (prevención e intervención) con personas y/o grupos, desde una línea normalizada o especializada, con objeto de generar cambios para la mejora y transformación social. Para estas labores, la creación de material audiovisual puede resultar de especial importancia en actividades de concienciación social y desarrollo de las competencias necesarias en el desempeño profesional del futuro educador social.

Una de las características más destacables de la actual Sociedad de la Información es la relevancia que las Tecnologías de la Información y la Comunicación (TIC) han asumido, siendo elementos de discriminación y exclusión en muchos casos en determinados contextos sociales (Helsper, 2008). Es en el contexto educativo, dónde las TIC proveen al profesor y alumno de un considerable número de herramientas y escenarios, utilizándose en procesos de implementación de estrategias didácticas, con el propósito de facilitar la construcción de conocimiento (Martínez-Palmera, Combita-Niño y De-La-Hoz-Franco, 2018). En este sentido, las universidades se están reformando debido a la incorporación de las TIC, particularmente a causa de la aparición y desarrollo de Internet 2.0. A su vez, el uso de ellas en los procesos de enseñanza-aprendizaje están cambiando la forma en que se realiza la docencia en la educación superior y sus recursos en línea representan una de las fuentes más comunes para el aprendizaje entre los estudiantes universitarios. 
En esta investigación se describe una actividad didáctica innovadora desde una perspectiva diacrónica, en la que se presenta el proceso de enseñanza para crear un repositorio audiovisual por el estudiante en el desarrollo de sus competencias digitales y vinculadas al perfil profesional de sus estudios universitarios como Educador Social. Asimismo, se creó una rúbrica como herramienta de evaluación, utilizándose para analizar la calidad de los vídeos y determinar los aspectos más positivos y aquellos en los que los estudiantes debían incidir para proporcionar mayor calidad técnica a sus producciones audiovisuales. Para ello, los ítems de la rúbrica se centraron en la evaluación aspectos del entorno didáctico y técnicos del material audiovisual.

\section{ANTECEDENTES DEL VÍDEO COMO RECURSO DIDÁCTICO}

Estudios como los de Ramos (1996) hablan del vídeo como recurso metodológico haciendo alusión por un lado, a su eficacia por estar relacionado especialmente con la forma en la que se llevaría a cabo su aplicación en el contexto de la clase y con la presencia de elementos significativos que indiquen una relación directa entre sus contenidos, el programa de la asignatura y quiénes lo imparten. Y por otro lado, su uso estaría determinado por su bajo coste o su facilidad de manejo, que le permitiría estar presente en distintos momentos del proceso educativo: como medio de observación, como medio de expresión, como medio de autoaprendizaje y como medio de ayuda a la enseñanza.

Investigaciones como las de Cabero (2007) afirman que el vídeo podría ser utilizado por el profesor por las potencialidades que ofrece, las cuales repercuten en la creación de nuevos entornos, tipos y facilidades para el aprendizaje; aspectos que van desde la modificación de la interacción comunicativa unidireccional entre profesor-alumno, hasta la reforma física-espacial-temporal de los escenarios de aprendizaje, sin olvidar las posibilidades que ofrecería para el autoaprendizaje y el aprendizaje cooperativo entre estudiantes de diferentes contextos físicos y culturales. Por tanto, es aquel que serviría para la educación y el logro de un objetivo previamente formulado. Y permitiría reflexionar sobre la puesta en marcha de un nuevo paradigma formativo y sobre como alcanzar el umbral de lo que debería ser una verdadera sociedad del conocimiento (Garrido, 2006).

\section{El vídeo como recurso didáctico en la educación superior}

Actualmente, la digitalización de la imagen audiovisual y el acceso a videocámaras desde los dispositivos móviles ha supuesto la apropiación de este tipo expresión por parte de los sectores masivos de la población. Ahora somos capaces de capturar, transmitir, almacenar y reproducir imágenes de una gran calidad, permitiendo abrir nuevos espacios para comunicarnos y desarrollar la creatividad. En este sentido, los audiovisuales como el vídeo digital han permitido que el estudiante deje de ser un agente receptor pasivo y pase a ser un educando activo dentro del proceso de aprendizaje. A su vez, estos, pueden considerarse como instrumentos didácticos relevantes para la creación de signos, significados y para la elaboración de conceptos (Cabero, Vázquez-Cano y López-Meneses, 2018).

Lo que se busca es comprender, explicar la realidad y crear valores, que constituyen las subjetividades generadas por experiencias y expectativas (Tobías, Duarte y Kemczinski, 2015). Además, su empleo puede formar parte de los entornos personales de aprendizaje utilizados en el proceso de e-learning en diversas áreas (Yellepeddi y Roberson, 2016; DelSignore, Wolbrink, Zurakowski y Burns, 2016) e implicaciones positivas para el alumnado, tanto a nivel de actitud, como de procedimientos y resultados (Kay y Kletskin, 2012).

Para Bartolomé (2008) las posibilidades para la creación, edición y publicación de vídeo constituyen una de las características más definitorias de la Web 2.0. Este hecho ha supuesto que el sistema de grabación y reproducción de imágenes y audios haya resurgido con fuerza como recurso educativo, además de estimular la construcción del conocimiento (Tobías, Duarte y Kemczinski, 2015). En este sentido diferentes autores (Fernández, 2019;Troncoso-Pantoja, Amaya-Placencia y Pincheira-Aguilera, 2019; Cabero, 2007) realizan distintas reflexiones vinculadas con las fortalezas y debilidades del vídeo (Tabla 1).

Asimismo, entre los usos educativos del vídeo digital, surge el de aprovechar una de las características más tradicionales de la tecnología educativa, ofrecer información y aprovechar el valor motivador de las imágenes en movimiento, no obstante está perspectiva ha sido refutada en otras investigaciones (Sonmez y HakverdiCan, 2012). Por su parte, el estudiante se halla inmerso en una sociedad caracterizada por la cultura de la imagen y, por ello, hay que analizar la comunicación, en este caso audiovisual, que se desarrolla en dicha sociedad. A su vez, cuando el alumnado tenga la posibilidad de comunicar creativamente sus experiencias e interprete los mensajes que sus compañeros han realizado, estará formando su propio juicio crítico frente a los medios masivos y a los mensajes que estos trasmiten (Sonmez y Hakverdi-Can, 2012). 
Tabla 1: Posibilidades y limitaciones para el uso del vídeo en la enseñanza (Fernández, 2019;Troncoso-Pantoja, AmayaPlacencia y Pincheira-Aguilera, 2019;Cabero, 2007).

\begin{tabular}{|l|l|}
\hline Posibilidades del medio videográfico & \multicolumn{1}{|c|}{ Limitaciones del medio videográfico } \\
\hline $\begin{array}{l}\text { Medio para la enseñanza (medio de presentación de información por } \\
\text { el docente, medio para la educación audiovisual, e instrumento para } \\
\text { que los estudiantes elaboren sus propios mensajes) }\end{array}$ & $\begin{array}{l}\text { Su producción por los docentes y } \\
\text { estudiantado, requiere de formación } \\
\text { específica }\end{array}$ \\
\hline Posibilidades de utilizarse en diferentes niveles educativos & $\begin{array}{l}\text { Se necesita de una mínima dotación } \\
\text { instrumental }\end{array}$ \\
\hline Impulsa la autonomía del estudiante & \\
\hline Herramienta de investigación psicodidáctica & $\begin{array}{l}\text { Limitaciones del "copyright" que no suelen } \\
\text { considerarse en los centros educativos }\end{array}$ \\
\hline $\begin{array}{l}\text { Mejora las capacidades de observación, análisis y reflexión } \\
\text { permitiendo controlar el propio aprendizaje }\end{array}$ & \\
\hline Instrumento de análisis de la realidad circundante & \\
\hline Potencia el aprendizaje activo y motivador & \\
\hline $\begin{array}{l}\text { Favorece la retroalimentación entre actores favoreciendo la } \\
\text { reutilización acorde con las necesidades académicas del estudiante }\end{array}$ & \\
\hline $\begin{array}{l}\text { Se convierten en materiales para las plataformas tecnológicas por lo } \\
\text { que impulsan la innovación de forma constante }\end{array}$ & \\
\hline
\end{tabular}

El contexto socioeducativo actual demanda un sujeto alfabetizado audiovisualmente, toda vez que una persona analfabeta tecnológicamente queda al margen de la red comunicativa que ofertan los audiovisuales. Además, el aplicar diversos sistemas simbólicos ayuda a potenciar habilidades cognitivas diversificadas en los estudiantes, crea entornos variados, de interaccion entre los partcipaantes, ricos para el aprendizaje y facilitadores del acercamiento a la información. Asimismo, en el libro blanco para el el título de Pedagogía y Educación Social, elaborado por la ANECA (2005) se contempla la utilización de las Tecnologías de la Información y la Comunicación (TIC) en el ámbito de estudio y del contexto profesional.La cual es interpretada por este organismo en los términos «es la capacidad para utilizar las TIC como una herramienta para la expresión y la comunicación, para el acceso a fuentes de información, como medio de archivo de datos y documentos, para tareas de presentación, para el aprendizaje, la investigación y el trabajo cooperativo» (ANECA, 2005, p. 143).

\section{Contexto y desarrollo de la experiencia didáctica}

Esta investigación se contextualizó en dos asignaturas: "Tecnologías de la Información y la Comunicación en Educación Social", correspondientes, al primer año de la titulación del Grado de Educación Social y Doble Grado de Educación Social. Y se realizó durante cinco cursos académicos comprendidos desde el curso académico 2013-2014 hasta el 2017-18 en la asignatura: "Nuevas Tecnología y Gestión de la información" que corresponde a la titulación de Trabajo Social de la Facultad de Ciencias Sociales de la Universidad Pablo de Olavide, de Sevilla (España) con una carga de 7,3 y 8,9 Créditos ECTS ("European Credit Transfer System"), respectivamente. Con respecto al programa de estudios, la asignatura de "Tecnologías de la Información y la Comunicación en Educación Social”, pertenece al área de Didáctica y Organización Educativa y se articula alrededor de diversos bloques de contenidos; nuestra experiencia didáctica se enmarcó en el segundo núcleo temático denominado: "Recursos didácticos-tecnológicos y su utilización educativa", en concreto al que correspondía con el Tema 5: "Los medios informáticos y el software social". El organigrama conceptual de los bloques de la asignatura quedan reflejados en la figura 1.

La experiencia innovadora universitaria de los cinco cursos académicos se desarrolla generalmente durante los meses de marzo y abril y consiste en el diseño, desarrollo y evaluación de un trabajo audiovisual relacionado con la prevención y sensibilización de problemas sociales (3-6 minutos). Se compone de dos partes, la Guía Didáctica del Material Educativo Multimedia (MEM) y elaboración de un e-Portafolio/diario (grupal, 4-8 estudiantes) sobre el proceso creativo del material educativo audiovisual. Respecto a la Guía Didáctica tienen las siguientes partes: 1) justificación teórica. Fundamentación teórica actualizada y relevante de la temática del Material Educativo Multimedia (MEM); 2) destinatarios del material audiovisual; 3) objetivos; 4) esquema de contenidos. Realizar un mapa conceptual de los conceptos que trata el recurso digital; 5) guion multimedia/storyboard del material educativo multimedia (MEM); 6) enlace del recurso digital (youtube, vimeo...); y 7) referencias bibliográficas y Webgrafía (mínimo 5 enlaces, explicados y relevantes, relacionados con la temática del MEM). 


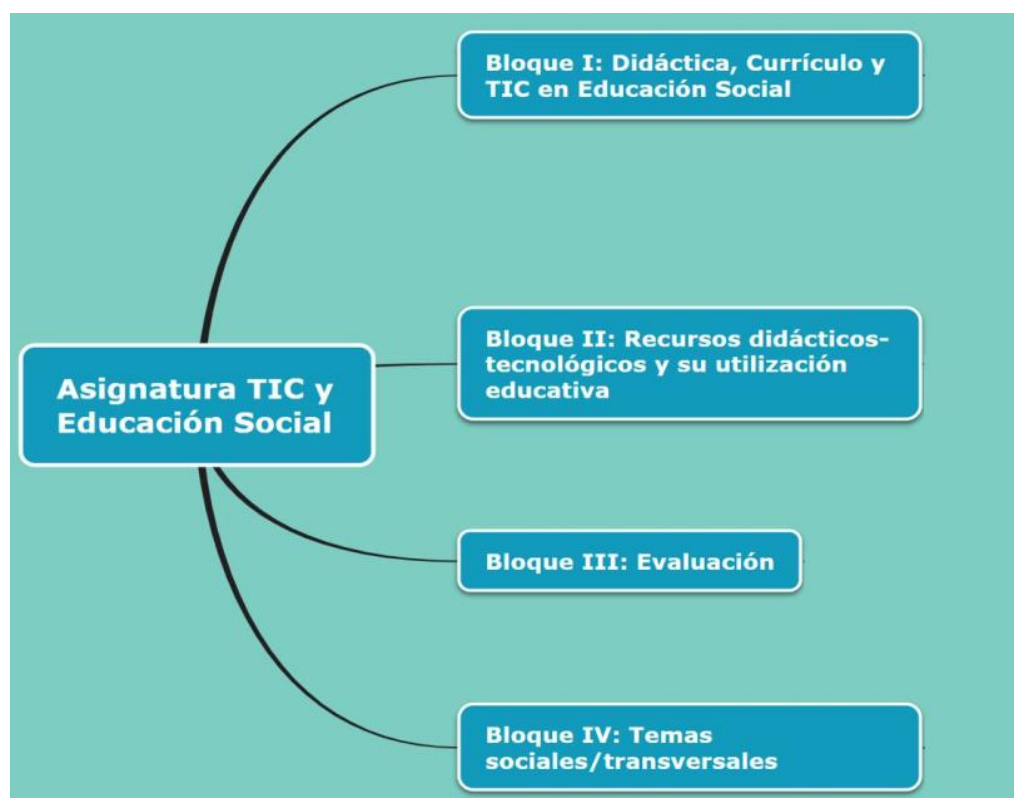

Fig. 1. Organigrama conceptual de los bloques de contenidos de la asignatura (www.mindomo.com/view.htm?m=6cd2bf5f280e4e7bb7cca11b77b8beb1).

La segunda tarea didáctica, era valorada sobre un $20 \%$ de la nota final de la asignatura, consistía en el envío de documentación relevante sobre las reflexiones, evidencias y progresos relacionados con la realización del proyecto multimedia. A través de comentarios (post) al edublog en portafolio electrónico universitario (LópezMeneses, 2011).

En este sentido, el portafolio o carpeta de trabajo del estudiante recopilaba de forma explícita las reflexiones, inquietudes, ideas, problemas encontrados durante el proceso del desarrollo del material didáctico audiovisual dentro y fuera del aula. También, se debía constatar las diferentes fuentes de información, los especialistas y educadores consultados, las tareas próximas, los borradores de progreso, las sugerencias y orientaciones expresadas en las tutorías por el profesor, las hipótesis de trabajo, las experiencias vividas relacionadas con el objeto de estudio/con la temática, entre otros. Es decir, el portafolio electrónico es un repositorio dinámico, una colección de documentos que evidencia la reflexión, el crecimiento profesional y el progreso del trabajo grupal (dentro y fuera de aula) respecto al desarrollo, en nuestro caso, del material didáctico audiovisual.

Para lograr los objetivos marcados a finales del mes de febrero se impartieron dos sesiones, de dos horas en el aula de informática para conocer las características más significativas de los servicios edublogs y de su aplicación en servicio de blogs: Blogger y las plataformas de Youtube y Vimeo para subir los videos de forma gratuita. Una vez terminada la obra audiovisual, cada estudiante la publicaba en su edublog personal. Posteriormente, el profesor de la asignatura recopilaba información de cada post y los subía a una web gratuita para la creación de un repositorio videográfico educativo con los videos elaborados por los estudiantes.

Hay que indicar que los vídeos para que tengan una orientación y enfoque educativo deben incentivar el pensamiento, despertando el interés del estudiante por los contenidos, además de ser globalizador, al permitir trabajar bajo diferentes perspectivas una misma temática, estimulando la discusión grupal. En este caso, en las sesiones teóricas se dedicaban a la visualización de diferentes vídeos elaborados por el estudiantado para avivar el interés y el diálogo constructivo sobre temáticas relacionadas con los ámbitos profesionales del educador social. En concreto, en el último curso se proyectaron los videos titulados: "La vida de una estudiante" donde se narraba la temática de la accesibilidad de una estudiante sorda en la universidad, sobre el envejecimiento activo: "Soledad en la tercera edad", o entorno a la marginación social con el vídeo educativo: "Entre cartones", donde los estudiantes experimentaron la angustia de pedir en la calle y, posteriormente grabaron personas marginadas y excluidas socialmente que viven en la calle. Es decir, se trataba de promover el desarrollo autónomo del estudiante para que fuera capaz de adquirir y actualizar conocimientos, destrezas y competencias a lo largo de su vida (Life Long Learning) en diferentes contextos de aprendizaje, formales y no formales, que contribuirían a su futuro desarrollo profesional y personal. Estudios como los de Sojka y Plch (2008) corroboran la idea del aprendizaje permanente, concluyendo que el uso de recursos didácticos electrónicos, entre ellos los vídeos, son una poderosa herramienta de visualización que ayuda a comprender la temática planteada, lo cual aumentando las posibilidades de aprendizaje y motivación del alumno. En nuestro caso muestra de esta motivación e incremento en los resultados de aprendizaje recae sobre los buenos resultados académicos obtenidos por los estudiantes de los cursos académicos en los que se realizo este estudio, de 2013 a 2018 (Tabla 2). 
Tabla 2. Resultados académicos de las titulaciones académicas de Trabajo Social, Grado de Educación Social y Doble Grado de Trabajo Social y Educación Social de 2013 a 2018.

\begin{tabular}{|l|c|c|c|c|c|c|}
\hline Titulación/año & N.P. & & Aprobados & Notables & Sobresalientes & MH \\
\hline Curso 2013-14. Grado de Trabajo Social & $3 \%$ & $4 \%$ & $4 \%$ & $12 \%$ & $77 \%$ & $0 \%$ \\
\hline Curso 2013-14. Grado de Educación Social & $4 \%$ & $5 \%$ & $6 \%$ & $15 \%$ & $70 \%$ & $0 \%$ \\
\hline $\begin{array}{l}\text { Curso 2014-2015. Grado de Educación } \\
\text { Social }\end{array}$ & $3 \%$ & $4 \%$ & $9 \%$ & $11 \%$ & $73 \%$ & $0 \%$ \\
\hline $\begin{array}{l}\text { Curso 2014-15. Doble Grado de Trabajo y } \\
\text { Educación Social }\end{array}$ & $3 \%$ & $5 \%$ & $7 \%$ & $13 \%$ & $72 \%$ & $3 \%$ \\
\hline Curso 2015-16. Grado de Educación Social & $4 \%$ & $1 \%$ & $2 \%$ & $8 \%$ & $82 \%$ & $3 \%$ \\
\hline $\begin{array}{l}\text { Curso 2015-16. Doble Grado de Trabajo } \\
\text { Social y Educación Social }\end{array}$ & $2 \%$ & $2 \%$ & $3 \%$ & $4 \%$ & $86 \%$ & $3 \%$ \\
\hline $\begin{array}{l}\text { Curso 2016-17. Grado de Educación Social } \\
\text { Curso 2016-17. Doble Grado de Educación }\end{array}$ & $4 \%$ & $3 \%$ & $5 \%$ & $8 \%$ & $80 \%$ & $0 \%$ \\
\hline $\begin{array}{l}\text { Social } \\
\text { Curso 2017-18. Grado de Educación Social }\end{array}$ & $0 \%$ & $9 \%$ & $2 \%$ & $26 \%$ & $60 \%$ & $3 \%$ \\
\hline $\begin{array}{l}\text { Curso 2017-18. Doble Grado de Trabajo } \\
\text { Social y Educación Social }\end{array}$ & $0 \%$ & $1 \%$ & $1 \%$ & $30 \%$ & $65 \%$ & $3 \%$ \\
\hline
\end{tabular}

También es de resaltar que la mayoría de los grupos de estudiantes, como se constata en sus portafolios de trabajo y en las sesiones teóricas y prácticas, utilizaban YouTube como plataforma para compartir vídeos. En este sentido, cabe mencionar que en la lista publicada cada año por Jane Hart (2017) del Centre of Learning \& Performance Technologies sobre las 200 mejores herramientas para el aprendizaje, en 2017 lidera la primera posición Youtube (Video sharing platform) y es una de las más aceptadas por los educadores. La relevancia de YouTube es notoria en relación a cualquier otra plataforma. Más de la mitad de los usuarios habituales de Internet declara ver vídeos a través de este canal diariamente, atribuyéndole característica tales como útil y práctica, convirtiéndose por tanto en el site más utilizado para visionado de contenidos audiovisuales (Ruiz y Sobrino, 2016). Asimismo, sirva de ejemplo "Química Analítica", grabación de material docente en vídeo de una duración inferior a 5-10 minutos, para el desarrollo y aplicación de materiales virtuales en español y en inglés. En el que más del $90 \%$ de los estudiantes consideró que la visualización previa del vídeo les resultaba sumamente útil a la hora de facilitar el trabajo de laboratorio (Palacios et al., 2016). En nuestro caso, a medida que pasaban los cursos académicos, hemos ido recortando el minutaje llegando como máximo a 5-10 minutos. Para ello, la mayoría del estudiantado editaban los videos educativos con el programa Movie Maker de Windows, acortándolos para cumplir con el requisito de la duración máxima de 5-10 minutos. En este sentido, esto puede implicar que el alumnado vaya adquiriendo habilidades y competencias digitales para manejar programas de edición de vídeos sencillos.

Por último, es de resaltar que las producciones audiovisuales del curso 2015-16 se publicaron en un libro titulado: "Innovar en las aulas universitarias", que está en acceso abierto, alojado en el repositorio institucional Olavide (RIO). A su vez, se utilizó una matriz de evaluación de la calidad de los vídeos por dimensiones que fue sometida a su validación mediante el juicio de experto y se calculó posteriormente los índices de fiabilidad de la misma. Una vez explicado el marco teórico que sustenta la investigación y el contexto didáctico de la misma, nos planteamos los siguientes objetivos: 1) crear y analizar un repositorio universitario videográfico para la sensibilización sobre problemas sociales en el área de Educación Social; 2) diseñar y validar una rúbrica de evaluación de los aspectos de ejecución técnica de los vídeos; y 3) evaluar la ejecución técnica de los vídeos creados por los estudiantes para mejorar su competencia digital en el desarrollo de los mismos.

\section{METODOLOGÍA}

La primera fase de la investigación se desarrolló conforme al diseño pedagógico explicado en el punto anterior y constituyó el corpus videográfico, consistente en 76 vídeos creados por estudiantes. Estos se categorizaron temáticamente y se procedió a su etiquetado anónimo para su evaluación. Posteriormente, la investigación se organizó en dos fases; una primera, que consistió en el diseño y evaluación de la rúbrica de evaluación de la calidad y desarrollo de los vídeos mediante expertos. La rúbrica de evaluación de los vídeos se sometió a un triple procedimiento de evaluación por juicio de 16 expertos conforme a las pruebas de V Aiken (1996), Razón de Validez de Contenido de Lawshe (1975) y coeficiente de congruencia de Osterlind (1989). En primer lugar, hallamos el Coeficiente de validez de contenido V de Aiken (Aiken, 1996). Esta es una de las técnicas que permite cuantificar la relevancia de cada ítem respecto de un dominio de contenido formulado por $\mathrm{N}$ jueces. Su valor oscila desde 0 hasta 1, siendo el valor 1 indicativo de un perfecto acuerdo entre los jueces 
respecto a la mayor puntuación de validez de los contenidos evaluados. Para ello, se eliminaron los ítems con una $V$ de Aiken de contenidos inferiores a 0.75 obtenidas con un nivel de confianza $p=0.99$ (Charter, 2003 ; Penfield y Giacobbi, 2004). En segundo lugar, se calculó el índice de Razón de Validez de Contenido de Lawshe (1975) que permite obtener un modelo específico de cuantificación de la representatividad de los ítems y la evidencia de que la definición semántica está bien representada en los ítems formulados. Para ello, la Razón de Validez de Contenido (RVC) debe ser igual o superior a 0.5823 (Tristan, 2008). En tercer lugar y ya comprobado el grado de acuerdo de los expertos y, por tanto, los ítems que permanecen y desaparecen de la prueba inicial, el siguiente paso fue verificar el grado de acuerdo de los jueces sobre la pertenencia de cada ítem a una de las dimensiones consideradas. Para ello, se procedió al cálculo del Grado de congruencia item-objetivo de Osterlind (1989). En una segunda fase, se analizaron las contribuciones de los estudiantes en forma de vídeo con la aplicación de la rúbrica creada. Para ello, participaron dos observadores y evaluadores independientes y se calculó el índice Kappa para comprobar la fiabilidad entre-jueces conforme a la siguiente fórmula (1) de (Brennan y Prediger, 1981), $\Sigma$ Pii es el porcentaje de acuerdo y $\Sigma$ Pi x Pi refleja la probabilidad de acuerdo (Holsti, 1969).

$\mathrm{K}=(\Sigma \mathrm{Pii}-\Sigma \mathrm{Pi} \times \mathrm{Pi}) /(1-\Sigma \mathrm{Pi} \times \mathrm{Pi})$

\section{RESULTADOS Y DISCUSIÓN}

En primer lugar, la codificación de los observadores cuantificó 79 vídeos, porque 3 de ellos ya no se encuentran disponibles en YouTube, por lo que los resultados finales los conforman 76 vídeos visibles en repositorio de videos de estudiantes (López-Meneses, 2012). Posteriormente, se procedió a generar la matriz de trabajo en la que se especificaba la temática, el curso y los estudiantes que habían creado cada vídeo.

Los resultados de las frecuencias temáticas muestran que el acoso escolar $(n=7)$, la inmigración ( $n=6)$, personas sin hogar $(n=6)$, la violencia de género $(n=6)$, y la discapacidad $(n=4)$, son las temáticas que presentan un mayor número de vídeos y que los estudiantes han entendido como prioritarias a la hora de dar una respuesta educativa y social. A continuación, en la figura 2, podemos ver la nube de palabras de las frecuencias temáticas de los 76 vídeos.

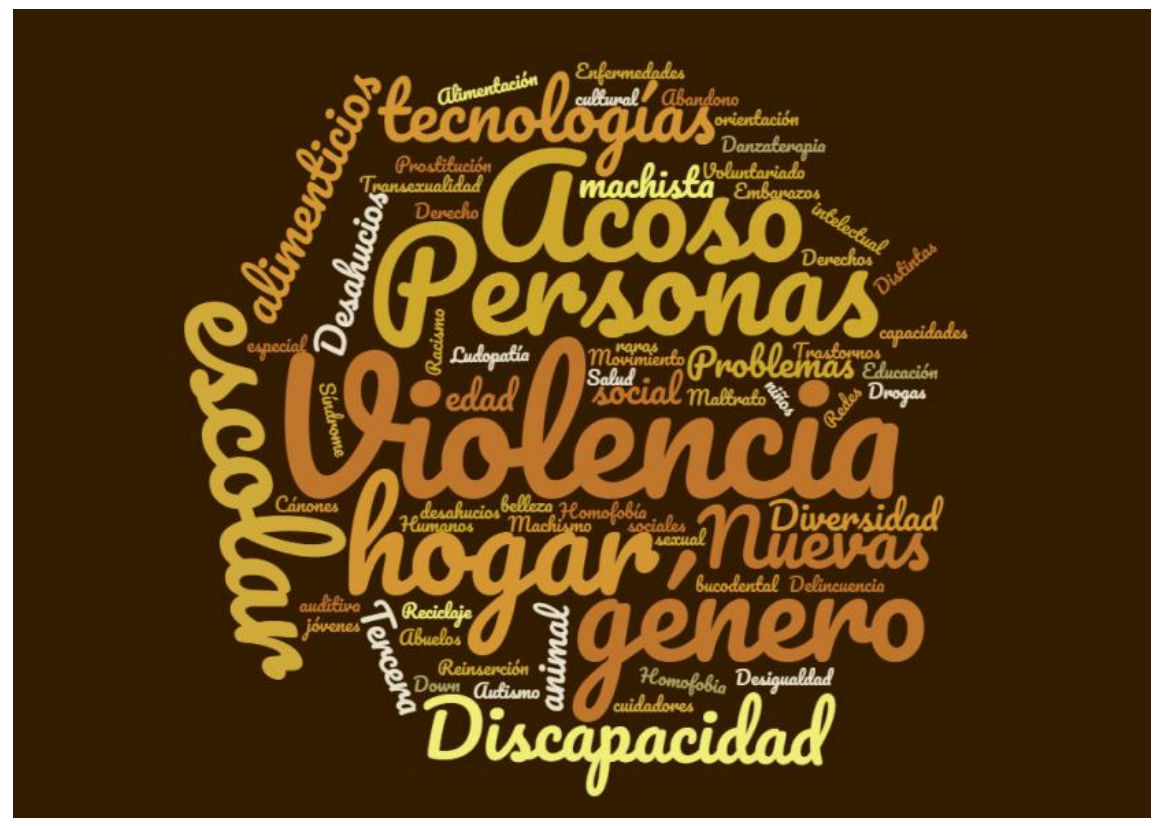

Fig. 2. Nube de palabras correspondiente a las temáticas del repositorio videográfico.

Posteriormente, se creó la rúbrica de análisis de la calidad técnica de los vídeos en base a una elaborada por el proyecto EDIA del Centro Nacional de Desarrollo Curricular en Sistemas no Propietarios (CEDEC), organismo dependiente del Ministerio de Educación, Cultura y Deporte a través del Instituto Nacional de Tecnologías Educativas y Formación del Profesorado (INTEF) y de la Consejería de Educación y Cultura del Gobierno de Extremadura (España). Rúbrica para evaluar un vídeo. Proyecto Cedec (VV.AA, 2016), e incorporando criterios de evaluación del programa de la asignatura que no podría englobarse en ninguno de las categorías de dicha rúbrica, creándose una propia para tal fin. En la tabla 3, mostramos los resultados de las tres pruebas para la conformación de la rúbrica final con los resultados estadísticos que validan cada ítem y su puntuación. 
Tabla 3: Cálculo de la fiabilidad de la rúbrica de evaluación (Aiken, 1996, Lawshe, 1975, Osterlind, 1989).

\begin{tabular}{|c|c|c|c|}
\hline Macrocategorías e Ítems & $\begin{array}{l}V \text { de } \\
\text { Aiken }\end{array}$ & Lawshe & $\begin{array}{c}l j k^{\prime} \\
N=15\end{array}$ \\
\hline Temática y Contenidos (3 puntos) & 0.91 & 0.85 & 0.87 \\
\hline $\begin{array}{l}\text { Fundamentación teórica es adecuada y pertinente con el ámbito de actuación } \\
\text { del Educador/a Trabajador/a Social. (1punto). }\end{array}$ & 0.89 & 0.87 & 0.85 \\
\hline $\begin{array}{l}\text { Se indican las finalidades educativas, los destinatarios y los contenidos (a } \\
\text { través de un esquema) del MEM. (1 punto). }\end{array}$ & 0.91 & 0.81 & 0.85 \\
\hline \multicolumn{4}{|l|}{$\begin{array}{l}\text { El Guión/ Storyboard del material audiovisual presenta un orden coherente y } \\
\text { cuenta con un inicio, desarrollo y conclusiones. (1punto). }\end{array}$} \\
\hline Aspectos técnicos (2 puntos) & 0.88 & 0.83 & 0.81 \\
\hline $\begin{array}{l}\text { Los elementos multimedia utilizados son coherentes con la temática } \\
\text { seleccionada y son relevantes para una mejor comprensión didáctica. }(0,5 \\
\text { punto). }\end{array}$ & 0.95 & 0.87 & 0.89 \\
\hline $\begin{array}{l}\text { Aparecen títulos de inicio y títulos de crédito finales, así como transiciones entre } \\
\text { diferentes partes. ( } 0,5 \text { punto). }\end{array}$ & 0.97 & 0.89 & 0.90 \\
\hline \multicolumn{4}{|l|}{ El Material Educativo Multimedia (MEM) es creativo e innovador. (1punto). } \\
\hline Portafolio digital (3 puntos) & 0.90 & 0.88 & 0.87 \\
\hline $\begin{array}{l}\text { El portafolio electrónico refleja evidencias de todas las fases de la elaboración } \\
\text { de un material audiovisual. (1punto). }\end{array}$ & 0.87 & 0.81 & 0.85 \\
\hline $\begin{array}{l}\text { El portafolio electrónico expresa con claridad las tareas próximas y las } \\
\text { dificultades encontradas. (0,5 punto). }\end{array}$ & 0.85 & 0.81 & 0.83 \\
\hline $\begin{array}{l}\text { Ha asistido a alguna tutoría para resolver dudas relacionadas con el trabajo } \\
\text { audiovisual. (1 punto). }\end{array}$ & 0.97 & 0.89 & 0.90 \\
\hline \multicolumn{4}{|l|}{$\begin{array}{l}\text { Estructura sintáctica adecuada con una redacción clara y uso de léxico amplio y } \\
\text { pertinente. (0,5 punto). }\end{array}$} \\
\hline Exposición del material audiovisual (2 puntos) & 0.91 & 0.89 & 0.88 \\
\hline $\begin{array}{l}\text { Voz clara, buena vocalización, entonación adecuada, postura corporal, atrae la } \\
\text { atención del público y mantiene el interés durante toda la exposición. ( } 0,5 \\
\text { punto). }\end{array}$ & 0.90 & 0.88 & 0.88 \\
\hline $\begin{array}{l}\text { Originalidad y creatividad en la exposición (la exposición se apoya en otros } \\
\text { elementos multimedia, etc.). (1punto). }\end{array}$ & 0.88 & 0.85 & 0.81 \\
\hline $\begin{array}{l}\text { Tiempo ajustado al previsto, con un final que retoma las ideas principales de la } \\
\text { exposición. (0,5 punto). }\end{array}$ & 0.88 & 0.85 & 0.81 \\
\hline
\end{tabular}

A continuación, mostramos un ejemplo de la identificación y categorización técnica de los vídeos creados por los estudiantes antes de proceder a su evaluación. Para ello, se tuvieron en cuenta los siguientes aspectos de cada vídeo: nombre del vídeo, minutaje, temática, número de integrantes que lo realizaron, sexo, plataforma (youtube o vimeo) y destinatarios potenciales (Tabla 4).

Tabla 4: Identificación y categorización técnica de los vídeos.

\begin{tabular}{|c|c|c|c|c|}
\hline Titulación/Curso académico & Grado en ES 13-14 & $\begin{array}{c}\text { Grado en ES 13- } \\
14\end{array}$ & $\begin{array}{c}\text { Grado en TS 13- } \\
14\end{array}$ & Grado en TS 13-14 \\
\hline Nombre del vídeo & $\begin{array}{l}\text { 4.Dependencia del } \\
\text { móvil }\end{array}$ & $\begin{array}{c}\text { 5.Madre } \\
\text { adolescente }\end{array}$ & $\begin{array}{l}\text { 5.Ludopatía y } \\
\text { Cleptomanía }\end{array}$ & $\begin{array}{l}\text { 6. Bullying ¿Y si } \\
\text { fueras tú? }\end{array}$ \\
\hline Minutaje & $3: 15$ & $6: 13$ & $6: 52$ & $3: 38$ \\
\hline Temática & Nuevas tecnologías & $\begin{array}{l}\text { Maternidad y } \\
\text { adolescencia }\end{array}$ & $\begin{array}{l}\text { Ludopatía y } \\
\text { Cleptomanía }\end{array}$ & Acoso escolar \\
\hline Número de integrantes & 4 & 5 & 5 & 4 \\
\hline Hombre/ Mujer & $4 \mathrm{M}$ & $1 \mathrm{H} / 4 \mathrm{M}$ & $1 \mathrm{H} / 4 \mathrm{M}$ & $1 \mathrm{H} / 3 \mathrm{M}$ \\
\hline Youtube o Vimeo & $\bar{Y}$ & $\bar{Y}$ & $\bar{Y}$ & $\bar{Y}$ \\
\hline Destinatarios potenciales & Toda la población & Toda la población & Toda la población & Toda la población \\
\hline Titulación/Curso académico & $\begin{array}{c}\text { Doble grado en ES Y } \\
\text { TS } 12-13\end{array}$ & $\begin{array}{c}\text { Doble grado en ES } \\
\text { Y TS 12-13 }\end{array}$ & $\begin{array}{c}\text { Grado en TS 12- } \\
13\end{array}$ & Grado en TS 12-13 \\
\hline Nombre del vídeo & 4.Adicción a las TIC & $\begin{array}{l}\text { 5. Reinserción de } \\
\text { jóvenes }\end{array}$ & 3.Desahucios & 4.El peso de tu vida \\
\hline Minutaje & 5:07 & $8: 58$ & $7: 52$ & $1: 56$ \\
\hline Temática & Nuevas tecnologías & Delincuencia & Desahucios & $\begin{array}{l}\text { Problemas } \\
\text { alimenticios }\end{array}$ \\
\hline Número de integrantes & 6 & 6 & 4 & 4 \\
\hline Hombre/ Mujer & $1 \mathrm{H} / 5 \mathrm{M}$ & $6 \mathrm{M}$ & $1 \mathrm{H} / 3 \mathrm{M}$ & $4 \mathrm{M}$ \\
\hline Youtube o Vimeo & $\bar{Y}$ & $\mathrm{Y}$ & $\bar{Y}$ & $\bar{Y}$ \\
\hline Destinatarios potenciales & Toda la población & Toda la población & Toda la población & Toda la población \\
\hline
\end{tabular}


Posteriormente se analizaron los vídeos por dos observadores conforme a la rúbrica creada y validada (Tabla 3 ), obteniendo un buen resultado en el índice Kappa ( $\mathrm{k}=.85 .58)$. En la tabla 5 , mostramos la frecuencia de unidades codificadas en el proceso.

Tabla 5: Resultados de la evaluación de la calidad delos vídeos conforme a la rúbrica empleada.

\begin{tabular}{|l|c|c|c|c|c|}
\hline \multicolumn{1}{|c|}{ Contenido } & $\begin{array}{c}\text { Totalmente de } \\
\text { acuerdo }\end{array}$ & De acuerdo & $\begin{array}{c}\text { Ni de acuerdo } \\
\text { ni en } \\
\text { desacuerdo }\end{array}$ & En desacuerdo & $\begin{array}{c}\text { Totalmente } \\
\text { en } \\
\text { desacuerdo }\end{array}$ \\
\hline $\begin{array}{l}\text { El vídeo presenta de manera clara y } \\
\text { concisa el trabajo realizado }\end{array}$ & $54(71 \%)$ & $10(13 \%)$ & $9(12 \%)$ & $2(3 \%)$ & $1(1 \%)$ \\
\hline $\begin{array}{l}\text { Presenta un orden coherente y cuenta } \\
\text { con un inicio, desarrollo y conclusiones }\end{array}$ & $53(70 \%)$ & 0 & $14(18 \%)$ & $8(11 \%)$ & $1(1 \%)$ \\
\hline La temática es original & $8(11 \%)$ & $30(39 \%)$ & $31(41 \%)$ & $7(9 \%)$ & 0 \\
\hline $\begin{array}{l}\text { Utiliza diversidad de material ilustrativo } \\
\text { (esquemas, gráficos, tablas, etc.) }\end{array}$ & 0 & $4(5 \%)$ & $10(13 \%)$ & $11(14 \%)$ & $51(68 \%)$ \\
\hline $\begin{array}{l}\text { El lenguaje utilizado es científico y } \\
\text { adecuado. }\end{array}$ & $1(1 \%)$ & $3(4 \%)$ & $60(79 \%)$ & $4(5 \%)$ & $8(11 \%)$ \\
\hline Diseño & $24(32 \%)$ & $11(14 \%)$ & $31(41 \%)$ & $8(11 \%)$ & $2(2 \%)$ \\
\hline La organización del vídeo es excelente & $23(30 \%)$ & $12(16 \%)$ & $31(41 \%)$ & $2(2 \%)$ & $8(11 \%)$ \\
\hline $\begin{array}{l}\text { Hay títulos que favorecen la } \\
\text { comprensión del tema por los posibles } \\
\text { espectadores }\end{array}$ & $64(85 \%)$ & $3(4 \%)$ & $6(8 \%)$ & $2(2 \%)$ & $1(1 \%)$ \\
\hline $\begin{array}{l}\text { Aparecen títulos de inicio y títulos de } \\
\text { crédito finales }\end{array}$ & $34(45 \%)$ & $15(19 \%)$ & $12(16 \%)$ & $8(11 \%)$ & $7(9 \%)$ \\
\hline $\begin{array}{l}\text { Aparecen transiciones entre diferentes } \\
\text { partes }\end{array}$ & $39(52 \%)$ & $17(22 \%)$ & $11(14 \%)$ & $5(7 \%)$ & $4(5 \%)$ \\
\hline $\begin{array}{l}\text { Predominio de planos sólo tres (plano } \\
\text { general o de paisaje, plano medio o } \\
\text { plano corto) }\end{array}$ & $11(14 \%)$ & $6(8 \%)$ & $3(4 \%)$ & $3(4 \%)$ & $53(70 \%)$ \\
\hline $\begin{array}{l}\text { Narrador en voz en off } \\
\text { Ambientación musical }\end{array}$ & $61(81 \%)$ & $8(11 \%)$ & $4(5 \%)$ & $1(1 \%)$ & $2(2 \%)$ \\
\hline El diseño es creativo e innovador & $7(9 \%)$ & $23(30 \%)$ & $30(40 \%)$ & $11(14 \%)$ & $5(7 \%)$ \\
\hline Elementos técnicos & $49(64 \%)$ & $10(13 \%)$ & $11(14 \%)$ & $5(7 \%)$ & $1(1 \%)$ \\
\hline El vídeo es de una gran nitidez & $23(30 \%)$ & $42(55 \%)$ & $5(7 \%)$ & $6(8 \%)$ & 0 \\
\hline \begin{tabular}{l} 
El volumen es el adecuado \\
\hline Los títulos son legibles
\end{tabular} & $45(60 \%)$ & $27(36 \%)$ & $2(2 \%)$ & $1(1 \%)$ & $1(1 \%)$ \\
\hline $\begin{array}{l}\text { No hay ningún problema técnico de } \\
\text { carácter grave. }\end{array}$ & $48(63 \%)$ & $22(30 \%)$ & $4(5 \%)$ & $2(2 \%)$ & 0 \\
\hline
\end{tabular}

En las figuras 3 y 4 sintetizamos los aspectos que muestran un mayor y menor cumplimiento de los aspectos técnicos conforme a la aplicación de la rúbrica y el acuerdo de los observadores y evaluadores de los vídeos. La evaluación de la calidad técnica y de ejecución de los vídeos muestran que hay áreas susceptibles de mejora como son: la creatividad, el lenguaje técnico y el narrado en off de los vídeos. Asimismo, se debe hacer hincapié en aspectos de diseño organizativo y de diseño (títulos y transiciones) que precisan una intervención docente que oriente y mejore el "microediting" de los vídeos.

\footnotetext{
Presenta un orden

coherente y cuenta con un inicio, desarrollo y conclusiones

Aparecen títulos de inicio y títulos de crédito finales

Ambientación musical

El vídeo es de una gran nitidez

Los títulos son legibles

No hay ningún problema técnico de carácter grave.
}

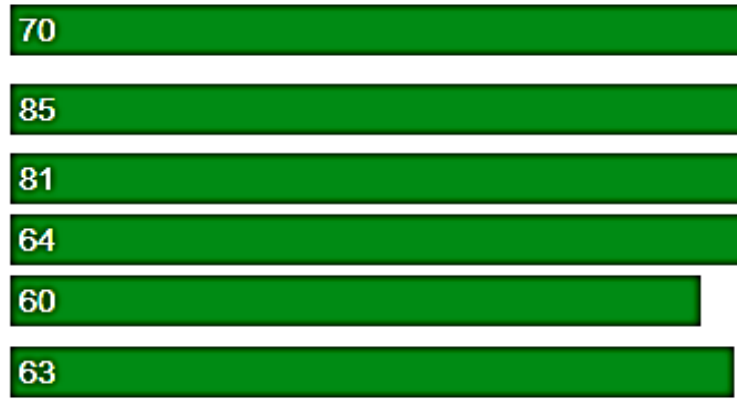

Fig. 3. Aspectos técnicos mejor valorados en el desarrollo de los vídeos. 


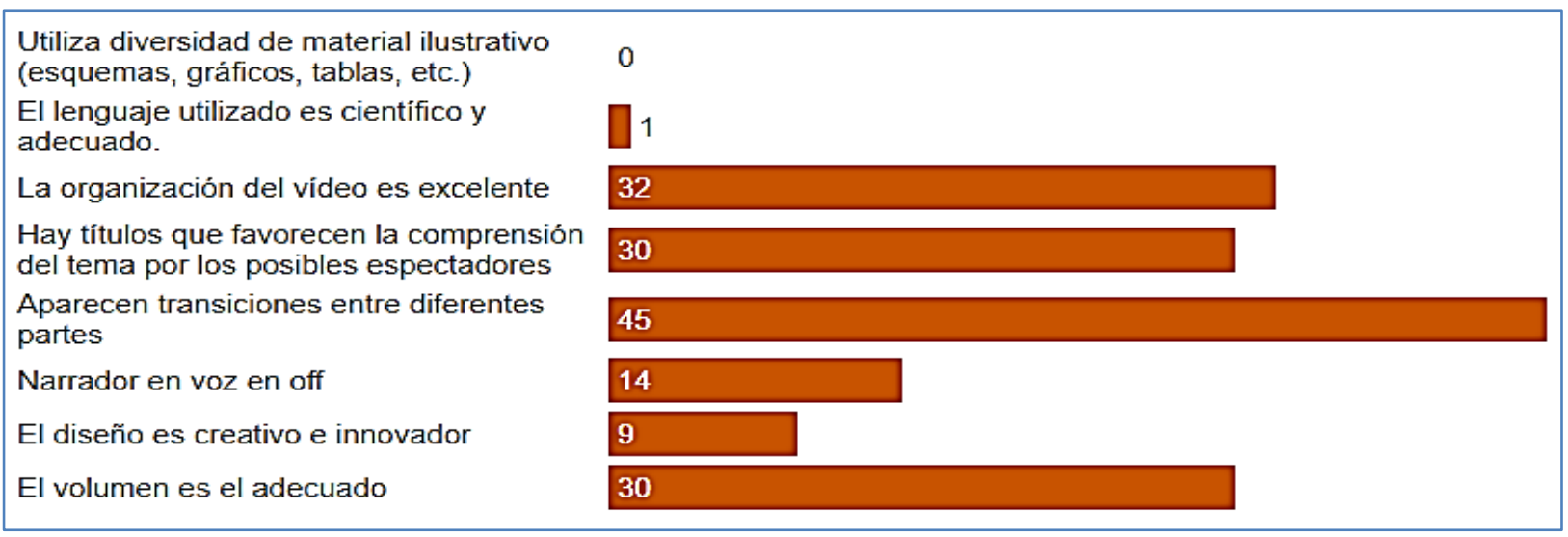

Fig. 4. Aspectos técnicos peor valorados en el desarrollo de los vídeos.

En la actualidad las universidades se están reformando debido a la incorporación de las tecnologías de la información (TIC), particularmente a causa de la aparición y desarrollo de Internet 2.0. Las tecnologías, al ser usadas como herramientas del conocimiento constructivista, entendido como aquel que se descubre y se construye. En el que el alumno cimienta su conocimiento a partir de su propia forma de ser, pensar e interpretar la información (Requena, 2008). Crean una experiencia diferente al proceso de enseñanzaaprendizaje tradicional, incentivan su manera de aprender; por lo tanto, ellos aprenden mejor y construyen su propio conocimiento. En este sentido, en concordancia con Tobías, Duarte y Kemczinski (2015), la intención educativa prioritaria de la praxis innovadora universitaria es la creación de un espacio virtual de intercambio de experiencias por medio de vídeos para mejorar los procesos de aprendizaje de los estudiantes universitarios. En este sentido, desde el grupo de investigación Eduinnovagogía ${ }^{\circledR}$ (HUM-971) continuamos buscando sinergias para que dicho repositorio de videos digitales construido por el estudiantado se vincule con otras instituciones internacionales de Educación Superior.

Coincidimos con Tobías, Duarte y Kemczinski (2015), que las posibilidades para la creación, edición y publicación de vídeo constituyen una de las características más definitorias de la Web 2.0, suponiendo este hecho un resurgir como recurso educativo y estimulante en la construcción de nuevos conocimientos. También, cabe mencionar respecto a los canales utilizados, que la red social Youtube, en nuestro caso, fue un recurso didáctico muy interesante y funcional al permitir presentar en clase los diferentes trabajos de los grupos de estudiantes y generar un debate moderado por ellos mismos. Asimismo, gracias a esta plataforma (utilizada por todos el alumnado, excepto un grupo que utilizó la plataforma VIMEO) los estudiantes universitarios subieron sus composiciones audiovisuales fácilmente y gratuitamente y accedió al visionado de una amplia variedad de vídeos de los otros cursos académicos en los que se mostraban diferentes situaciones y problemáticas sociales, además de posibilitar la génesis de un repositorio de buenas prácticas prosociales que servirán a futuras promociones de egresados. En este sentido, consideramos que YouTube puede constituirse en un interesante recurso didáctico gratuito en la Educación Superior con un gran potencial pedagógico para favorecer entornos motivadores y dinamizadores para el aprendizaje cognitivo y colaborativo entre los estudiantes y entre éstos y el docente, además de contribuir a la democratización de pensamientos y a la difusión mundial de experiencias didácticas para la comunidad global educativa.

La creación y difusión de vídeos educativos entronca directamente con el desarrollo de competencias digitales conforme a los requerimientos europeos del marco de referencia para las competencias digitales (DigCompEdu). En concreto, con el Área 2 "Creación y distribución de recursos digitales" y las competencias asociadas a estas actividades: 2.1. Desarrollo de contenidos digitales. 2.2. Integración y reelaboración de contenidos digitales y 2.3. Derechos de autor y licencias. El desarrollo de esta actividad audiovisual no solo permitía el desarrollo de tareas de creación, sino de conocimiento para desarrollar contenidos digitales respetando los derechos de autor, las licencias y la protección de datos personales e identidad digital. Asimismo, la vinculación y mejora de las competencias digitales, se ha asociado a la sensibilización del estudiante universitario ante la intervención en temáticas sociales, que como educadores sociales, pueden acometer. En este sentido, se muestra en la figura 5, el análisis de la percepción del estudiante sobre los ámbitos sociales de intervención susceptibles de ser mejorados (13) después del desarrollo de la actividad propuesta, mediante un análisis de mineria de texto aplicado a sus opiniones en los e-portafolios y la representación en grafo de las principales relaciones. Las 13 temáticas identificadas tienen grados de intermediación superiores a 3, lo que demuestra que al menos tres estudiantes han identificado relaciones entre los semas analizados. 


\begin{tabular}{|lrrrrrrr|}
\hline node & degree & frequency & beweenness & topic & conductivity & locality & diversivity \\
social & 11 & 4 & 0.016883 & 6 & 15.3 & 1 & 42.2 \\
violencia & 6 & 2 & 0.025974 & 1 & 43.3 & 0 & 129.9 \\
genero & 6 & 3 & 0.039935 & 1 & 66.6 & 0 & 133.1 \\
salud & 5 & 2 & 0.026299 & 8 & 52.6 & 0 & 131.5 \\
trastornos & 4 & 1 & 0.000325 & 3 & 0.8 & 5 & 3.2 \\
personas & 4 & 1 & 0.000325 & 3 & 0.8 & 5 & 3.2 \\
mental & 4 & 1 & 0.004058 & 8 & 10.1 & 0 & 40.6 \\
mediación & 4 & 1 & 0.004058 & 8 & 10.1 & 0 & 40.6 \\
maltrato & 4 & 1 & 0.011364 & 1 & 28.4 & 0 & 113.6 \\
integración & 4 & 2 & 0.001948 & 2 & 4.9 & 1 & 9.7 \\
infantil & 4 & 1 & 0.003247 & 1 & 8.1 & 0 & 32.5 \\
hogar & 4 & 1 & 0.001299 & 3 & 3.2 & 1 & 13.0 \\
bullying & 4 & 1 & 0.000325 & 1 & 0.8 & 5 & 3.2 \\
sociocultural & 3 & 1 & 0.000325 & 4 & 1.1 & 3 & 3.2 \\
\hline
\end{tabular}

Fig. 5: Temáticas de intervención social.

En última instancia, las nuevas tendencias tecnológicas emergentes son recursos muy valiosos para la construcción del conocimiento en los procesos de aprendizaje, propiciando la reformulación de metodologías socioconstructivistas e investigadoras y para el desarrollo sostenible de la ciudadanía global.

\section{CONCLUSIONES}

De la información extraída a través de este estudio, de su discusion y analisis, se pueden formular las siguientes conclusiones:

Las competencias del docente se convierten en una demanda y objetivo prioritario en la Educación Superior al estar inmerso en la sociedad de la información y la comunicación.

Se ha ofrecido un cambio en la ejecución de la docencia al convertirse los recursos realizados por el alumnado en fuente de aprendizaje entre los estudiantes universitarios, al abrir nuevos espacios para que puedan comunicarse y desarrollar su creatividad.

Se ha evidenciado que los estudiantes precisan ser capaces de interpretar el contexto social y profesional vinculado a sus estudios universitarios, lo cual se facilita a través de la complementariedad llevada a cabo entre los conocimientos teóricos y prácticos.

Está complementariedad en su faceta práctica ha quedado generosamente reforzada con la creación del material audiovisual por parte del alumnado, demostrándose que se han establecido mejoras en el proceso de enseñanza-aprendizaje, como puede comprobarse en los resultados académicos.

La metodología socioconstructivista e investigadora utilizada ha despertado la motivación y ha aumentado el compromiso del estudiante, siendo una de las principales razones el sentirse protagonistas activos de su proceso de aprendizaje, al compartir entornos cooperativos, colaborativos y conseguir resultados competenciales.

El material creado se ha convertido en una pieza clave en el desarrollo de competencias y actividades de concienciación social, necesarias en el desempeño profesional como futuros educadores sociales.

El permitirles acceder a aquel material que le despierta interés, curiosidad o dificultad, tantas veces como sea necesario, garantiza su motivación y aprendizaje.

Se puede considerar estos materiales como instrumentos didácticos relevantes para la creación de signos, significados y para la elaboración de conceptos. 


\section{REFERENCIAS}

Aiken, L., Tests psicológicos y evaluación. México: Prentice Hall (1996).

Abarca, M. y Soto, X., Buenas prácticas evaluativas: análisis de experiencias en universidades chilenas. Evaluación del aprendizaje en innovaciones curriculares de la educación superior, 83 (2014).

ANECA., Libro Blanco de Grado en Pedagogía y Educación Social (vol. 1). Madrid: Agencia Nacional de Evaluación de la Calidad y Acreditación (2005).

Bartolomé, A., Web 2.0 and new learning paradigms. ELearning papers, 8, 1-10 (2008).

Brennan, R.L. y Prediger, D.J., "Coefficient Kappa: Some Uses, Misuses, and Alternatives". Educational and Psychological Measurement, 41, 687-699 (1981).

Cabero, J., Nuevas tecnologías aplicadas a la educación. Madrid: McGraw-Hill (2007).

Cabero, J., Vázquez-Cano, E., y López-Meneses, E., Uso de la Realidad Aumentada como Recurso Didáctico en la Enseñanza Universitaria. Form. Univ., 11(1), 25-34 http://dx.doi.org/10.4067/S0718-50062018000100025 (2018).

Charter, R.A., A breakdown of reliability coefficients by test type and reliability method, and the clinical implications of low reliability. Journal of General Psychology, 130(3), 290-304 (2003).

DelSignore, L., Wolbrink, T., y otros dos autores. Test-enhanced e-learning strategies in Postgraduate Medical Education: A randomized cohort study. J. Medical Internet Research, 18 (11), 1-9. https://doi.org/10.2196/jmir.6199 (2016)

Fernández, L.M.P., El vídeo análisis: un nuevo enfoque para la didáctica de la interpretación. MonTI. Monografías de Traducción e Interpretación, (11), 263-279 (2019).

Garrido, M. F., El reto del cambio educativo: nuevos escenarios y modalidades de formación. Educar, (38), $243-258$ (2006).

Hart, J., Top 200 tools for learning 2017. Recuperado de http://c4lpt.co.uk/top100tools/ (2017).

Helsper, E., Digital inclusion: an analysis of social disadvantage and the information society. Department for Communities and Local Government. (2008).

Holsti, O. R., Content analysis for the social sciences and humanities. MA: Addison-Wesley (1969).

Kay, R. y Kletskin, LI., Evaluating the use of problem-based vídeo podcasts to teach mathematics in higher education. Computers \& Education, 59, 619-627. https://doi.org/10.1016/j.compedu.2012.03.007 (2012).

Lawshe, Ch.H., A quantitative approach to content validity. Personnel Psychology, 28, 563-575 (1975).

López-Meneses, E., Portafolio electrónico universitario. Recuperado de: http://diariotrabajosocial.blogspot.com (2011).

Martínez-Palmera, O, Combita-Niño, H. y De-La-Hoz-Franco, E., Mediación de los Objetos Virtuales de Aprendizaje en el Desarrollo de Competencias Matemáticas en Estudiantes de Ingeniería. Formación Universitaria, 11(6), 6374. https://dx.doi.org/10.4067/S0718-50062018000600063 (2018).

Osterlind, S. J., What Is Constructing Test Items? (pp. 1-16). Springer Netherlands (1998).

Palacios, J.M., y otros 5 autores, Desarrollo y aplicación de materiales virtuales en español y en inglés para un curso de Química Analítica International Journal of Educational Research and Innovation (IJERI), 5, 55-71 (2016).

Penfield, R. D. y Giacobbi, P. R., Jr., Applying a score confidence interval to Aiken's item content-relevance index. Measurement in Physical Education and Exercise Science, 8(4), 213-225 (2004).

Requena, S. H., El modelo constructivista con las nuevas tecnologías: aplicado en el proceso de aprendizaje. RUSC. Universities and Knowledge Society Journal, 5(2), 26-35. (2008).

Ruiz, F. J. M. y Sobrino, M. Á. O., El vídeo como soporte en la narrativa digital del Branded Content y los productos audiovisuales en las plataformas online. Poliantea, 12(22), 85-116. (2016).

Sojka, P., y Plch, R. , Technological challenges of teaching mathematics in a blended learning environment, International Journal of Continuing Engineering Education and Life Long Learning, 18(5-6), 657-665 (2008).

Sonmez, D. y Hakverdi-Can, M., Videos as an Instructional Tool in Pre-Service Science Teacher Education. Eurasian Journal of Educational Research, 46, 141-158 (2012).

Tristan, A., Modificación al modelo de Lawshe para el dictamen de validez de contenido de un instrumento objetivo. Avances en Medición. 6(1), 37-48 (2008).

Tobías, M.Á., Duarte, M.C. y Kemczinski, A., A Digital Repository of Filmic Content as a Teaching Resource. Comunicar, 44, 63-71. https://doi.org/10.3916/C44-2015-07 (2015).

Troncoso-Pantoja, C. A., Díaz-Aedo, F., Amaya-Placencia, J. P., y Pincheira-Aguilera, S., Elaboración de videos didácticos: un espacio para el aprendizaje activo. FEM: Revista de la Fundación Educación Médica, 22(2), 91-92 (2019).

VV.AA., Rúbrica para evaluar un vídeo. Proyecto Cedec. Recuperado de: https://es.slideshare.net/cedecite/rbrica-paraevaluar-un-vdeo-resumen-de-un-proyecto (2016).

Yellepeddi, V., y Roberson, Ch., The use of animated videos to illustrate oral solid dosage form manufacturing in a pharmaceutics course. American Journal of Pharmaceutical Education, 80 (8), 1-10. (2016). 